\title{
Stimulus Recognition in Aural Paired-Associate Learning ${ }^{1}$
}

\author{
Edwin Martin \\ University of Michigan, Ann Arbor, Michigan
}

\begin{abstract}
Aural paired-associates were learned by the study-test method. On test trials, the study-trials stimuli were randomly intermixed among new stimuli and the Ss, prior to responding, had to indicate whether the presented stimulus was old or new. It was found that correct responding was contingent upon stimulus recognition, and that correct responding given nonrecognition of the stimulus was at the chance level.
\end{abstract}

In attempting to explain how it is that a given stimulus $\mathrm{S}$ comes to control behavior in the sense that the occurrence of $S$ is the occasion for response $R$, one determines almost immediately that the idea of a direct, one-to-one relationship between $S$ and $R$ cannot suffice. Subsequent to the presentation of $S$, but prior to the overt R, Ss make responses other than $R$. For example, it is evident that in many learning situations, upon presentation of $S$, Ss selectively focus on only certain aspects of $S$ and that frequently they code or recode those perceived aspects in various ways so as to obtain a facilitory mediating link between $\mathrm{S}$ and $\mathrm{R}$ (Lawrence, 1963; Shepard, 1963; Underwood, 1963).

The intervening response of concern in the present research is the recognition response. That such a response might exist as an integral component of learned behavior is suggested by the fact that Ss, when asked, can sometimes successfully report whether or not the $S$ of the moment

${ }^{1}$ This research was supported by the Advanced Research Projects Agency, Department of Defense, monitored by the Air Force Office of Scientific Research, under Contract No. AF 49(638)-1235 with the Human Performance Center, Department of Psychology. The data were collected by Robert H. Savage. is the same as an earlier S (Shepard and Teghtsoonian, 1961). This can only mean that a common response of some sort was made on both occasions and that $S$ s are capable of detecting such communality between responses. The implication for the learning process is: if a $S$ does not make essentially the same perceptual response to the $\mathrm{S}$ of the moment as he made to that $\mathrm{S}$ earlier, he can hardly treat the $\mathrm{S}$ of the moment as the signal for the response $\mathrm{R}$ he learned to make to $\mathrm{S}$ earlier. Thus, it seems appropriate to say that in order for a consistent, learned behavior form $\mathrm{R}$ to become the more or less certain consequence of the stimulus event $S$, the $S$, who is known to be capable of a multitude of intermediary selecting, coding, and other facilitative responses, must perforce come to make an intermediary response that in some sense is invariant over trials. Recognition may be identified with this stable perceptual mediator. That the learned response $\mathrm{R}$ cannot itself serve this function is demanded by evidence of recognition where no such $R$ is given by the S (Shepard and Teghtsoonian, 1961), and by the results of the present experiment.

The experiment to be reported was designed to test the hypothesis that in order for the stimulus $S$ to elicit the response $R$, 
the $S$ must make an intervening recognition response to $S$. The likelihood of a correct response given recognition or nonrecognition of the stimulus was observed in an aural paired-associate task where recognition was made difficult, and hence probabilistic, experimentally. In addition, stimulus meaningfulness (M) was manipulated with the idea that if the two processes, recognition and association formation, proved separable, the locus of the stimulus-M effect might be uniquely identifiable.

\section{Method}

Materials and Procedure. Each $S$ was given 12 study-test trials on a single paired-associate list of eight trigram-number pairs. Within the list, four of the trigrams were low-M CCCs and four were high-M CCCs, averaging to $8 \%$ and $80 \%$ association value (Witmer, 1935), respectively. Intralist stimulus similarity was kept as minimal as seemed possible. The responses were the digits 1 through 8. Four lists were drawn up by matching each of two different (but ostensibly equivalent) sets of eight trigrams with each of two random permutations of the eight digits.

On study trials, the $S$ listened silently to the pairs as they were presented aurally at a 2-sec rate. The stimuli were spelled, the responses said, in $E$ 's ordinary, conversational tone of voice. On each of the 12 study trials, the eight pairs were presented in a new random order.

Alternating with the 12 study trials were 12 test trials. On each test trial, 24 trigrams were presented. Of these, eight were the stimuli repeated on study trials and 16 were new trigrams that had never appeared on any previous trial, study or test. Half of these 16 filler trigranis were low-M CCCs, the other half high-M CCCs. The study-trials trigrams and the filler trigrams were intermixed in a random order that was different on every test trial. The presentation rate was one trigram every $6 \mathrm{sec}$.

On test trials, upon hearing a trigram, the $S$ first had to press one of two buttons, one marked "old" and the other marked "new," thereby indicating whether or not he recognized the trigram as one that occurred on study trials. He then had to say aloud one of the digits 1 through 8 : if he thought a given trigram was new, he was to say any one of the eight digits that came to mind; if he thought it old, he was to say the digit that went with it on study trials.

The Ss were instructed that the same eight pairs would occur on every study trial and that on each test trial all trigrams not from the studytrials list would be genuinely new. All materials were pre-recorded on tape and presented to the $S$ aurally through earphones. The intertrial interval was $5 \mathrm{sec}$, during which time the $S$ was alerted as to whether the upcoming trial was to be a study or a test trial.

Subjects. The Ss were 32 University of Michigan junior and senior females who volunteered for paid participation. Of these, eight were assigned to each of the four lists in such a way that after every fourth $S$ each list was represented by an equal number of $S$ s.

\section{Results and Discussion}

Recognition. A trial-by-trial summary of the recognition button-pressing data is shown in Fig. 1. Proportion recognitions (calling old stimuli "old") proceeds to a higher asymptote for high-M stimuli than for low-M stimuli. Over trials, high-M stimuli prove significantly easier to recognize than low-M stimuli $(z=6.56, p<$

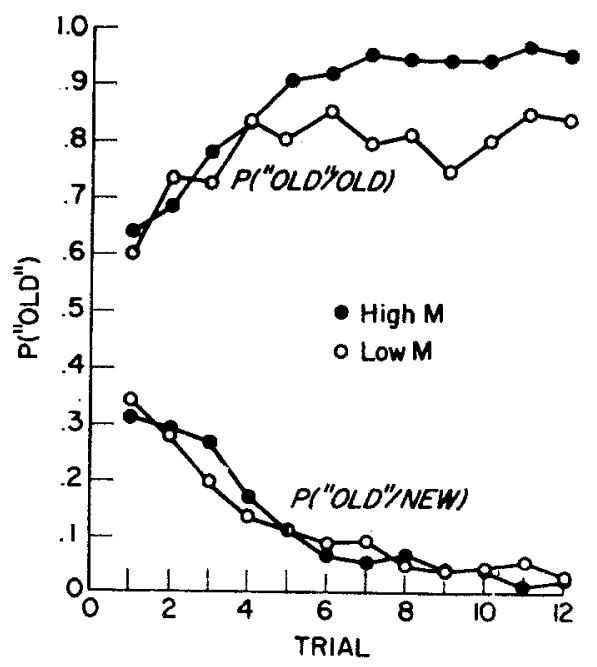

FrG. 1. Proportion "old" responses when stimuli are old (recognitions) and new (false positives). 
$.001)^{2}$. On the other hand, the proportion of false positives (calling new stimuli "old") is clearly not affected by $\mathrm{M}(z=$ $.12, p=.90$ ).

The results of the recognition buttonpressing task indicate that whereas $M$ is not a factor in the $S$ 's ability to correctly identify new stimuli, $M$ is a significant factor in his ability to recognize old stimuli. Two points regarding these findings might be considered. First, there is the possibility that were $\mathrm{M}$ to be manipulated between $S s$, instead of within $S$ s as in the present experiment, the $M$ effect would not materialize. This conjecture is based on the idea that in a within-Ss design, Ss can categorize verbal units according to $M$ and respond differentially to the separate categories. Second, it may seem curious that $M$ should have a significant effect on the proportion of recognitions but no effect on the proportion of false positives. If low-M stimuli are more difficult to recognize as old when they are old, then why should they not be more difficult to reject as new when they are new?

Evidence relevant to these matters comes from a recognition-memory experiment conducted by Martin and Melton (1967). The Shepard-Teghtsoonian (1961) technique was used, and three levels of $\mathrm{M}$ and two types of materials, CCCs and CVCs, were studied in a completely between-Ss design. The significant role of $M$ in recognition memory reported in the present study was substantiated, thereby eliminating the possibility that the $M$ effect is only a within-Ss phenomenon. However, for both CCCs and CVCs, low- $M$ units induced considerably higher false-positive rates than did high-M units. Therefore, while a generality seems in order regarding the relationship between $M$

\footnotetext{
2 The standard. $z$ test for the difference between two proportions (e.g., Walker and Lev, 1953, p. $77 f)$.
}

and the proportion of correct recognitions, the effect of $M$ on the incidence of false positives is more complicated and will require further experimental and theoretical attention.

Learning. A trial-by-trial representation of the correct-response (CR) data is shown in Fig. 2. These points are based on test-trial responses to the study-trials, or repeated, stimuli. Proportion CRs given stimulus recognition increases steadily over trials. Given stimulus recognition, the

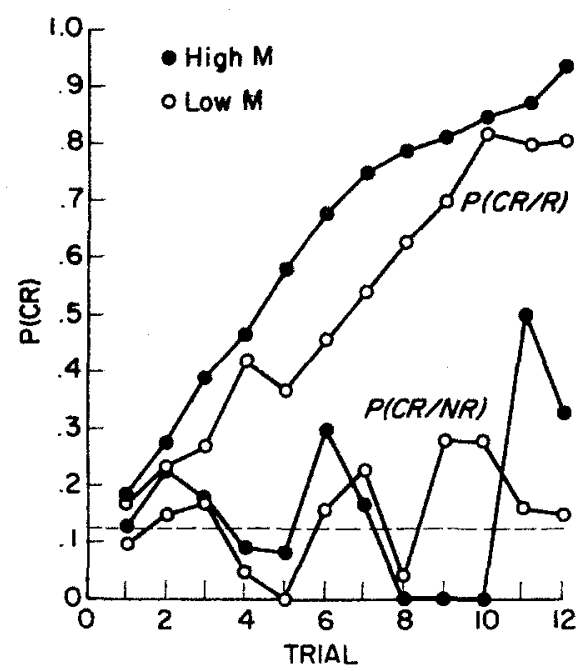

FIG. 2. Proportion correct responses (CR) given recognition $(R)$ and nonrecognition (NR) of highand low-M stimuli. The dashed line is the chance level.

effect of stimulus $M$ is significant ( $z=$ $6.60, p<.001)$. When the stimulus is not recognized, proportion CRs does not differ from the .125 chance level $(z=1.35$, $p=.18$ for high $M ; z=1.21, p=.23$ for low $M$ ) and $M$ is no longer a factor $(z=$ $.36, p=.72)$. The increasing irregularity of the $\mathrm{P}(\mathrm{CR} / \mathrm{NR})$ curves is due to the rapidly decreasing frequency of nonrecognitions.

With respect to the finding that given nonrecognition of the stimulus the likelihood of a CR is not different from chance, 
there are two interpretations that can be fairly safely eliminated. The first has to do with the possibility that stimulus recognition is not necessary for production of a $\mathrm{CR}$ and that the obtained results arose because the $S$ s adopted certain response strategies, for example, always giving a particular single response whenever a stimulus is judged new, thereby overriding whatever association might have been activated by that stimulus. An examination of the individual protocols revealed that only three out of the 32 Ss systematically responded with a same digit whenever they called an old stimulus new. If for each $S$ the number of times he called an old stimulus new is located on a horizontal axis, and if against this number is plotted the number of different digits he used as responses over those occasions, it turns out that 29 of the Ss give points sufficiently close to the values expected under an independent-guessing hypothesis (Riordan, 1958, p. 92) to conclude that they were in fact guessing freely. Thus it seems fair to say that response strategies (detectably more rational than choosing randomly) were not in general use.

The second interpretation that can be eliminated is that the stimuli that were not recognized are stimuli to which associating responses is difficult, which means simply that the lower curves in Fig. 2 represent especially difficult $S-R$ associations. If this were true, then certainly an $M$ effect would be apparent. But more compelling evidence can be obtained by calculating the proportion CRs, for both recognition and nonrecognition of the stimulus, where the number of preceding CRs is controlled. For example, consider all entries in the protocols of the $32 \mathrm{Ss}$ jointly satisfying the conditions that each entry (a) represents a recognition of the stimulus presented and (b) is preceded by exactly $X$ CRs. To facilitate exposition, let $X=2$. Among the high-M protocols, there were 161 stimulus events that were recognized and that followed exactly two previous CRs; 114 of them resulted in a CR. Thus for high-M stimuli, the proportion CRs after two previous CRs is .71, providing the stimulus is recognized. If the stimulus is not recugnized, proportion CRs after two previous CRs turns out to be only .14.

The proportion-CR data for $X=0,1$, . . ., 8 are plotted in Fig. 3. The last few

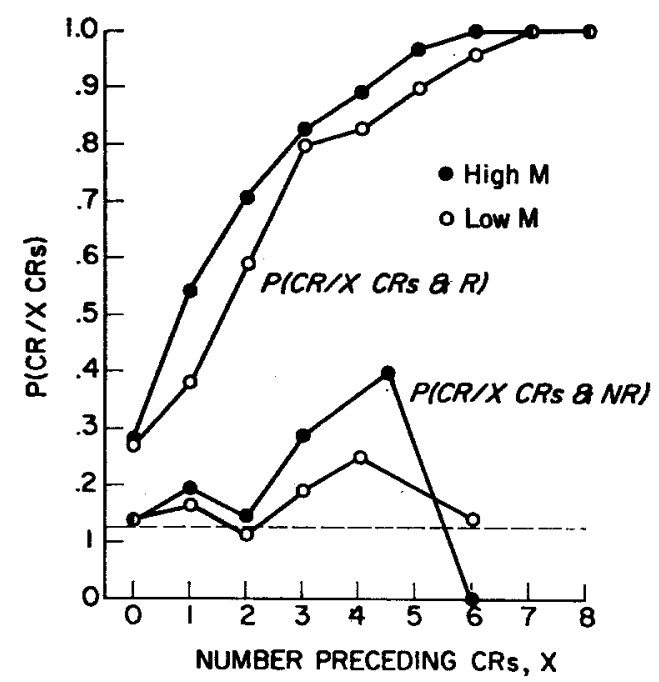

Fig. 3. Proportion correct responses (CR) given $X$ preceding CRs when high- and low-M stimuli are recognized $(R)$ and not recognized (NR). The dashed line is the chance level.

data points for the $\mathrm{P}(\mathrm{CR} / X$ CRs and NR) curves represent averages over adjacent values of $X$; this was done to combat the unreliability of proportions based on very small frequencies (no point represents less than five data). Thus when the preceding-CR history is controlled, that is, when the extent of prior learning is controlled, elicitation of a $\mathrm{CR}$ remains contingent upon recognition of its stimulus.

Thus the following variation on the hypothesis proffered at the outset appears to 
be acceptable: regardless of the current status of an S-R association, the activation of that association, and hence the occurrence of the response event $R$, has as a necessary antecedent recognition of the stimulus event $\mathrm{S}$. The utilization of an associative connection apparently requires a particular perceptual response to the stimulus member of that association, a perceptual response that consistently causes the $S$ to regard that stimulus as the same from one occasion to the next. There is little doubt that all Ss clearly heard all stimuli as they came over the earphones. Each $S$ therefore made a perceptual response of some sort to each stimulus. That these perceptual responses were variable, especially in early trials, is evidenced by the number of nonrecognitions. That this variability is preclusive of consistent correct responding is evidenced by the positive correlation between number of recognitions and number of CRs over trials (compare Figs. 1 and 2), but more importantly by the contingency of CRs on recognitions.

It is not implausible to suppose that a major portion of the paired-associate task is taken up with the establishment of consistent perceptual responses on which to base overt responses. The present writer sees the formation of such mediating responses as more difficult to low-M stimuli than to high-M stimuli, and distinct from the association-formation process that makes the perceived stimulus the occasion for a given overt response.

In a previous paper (Martin, 1966), which concerned itself with how stimulus $M$ affects paired-associate learning, it was hypothesized that it is $M$ in the sense of number of associations, as opposed to $M$ in the sense of serial integration, that determines rate of association formation be- tween a stimulus and a response. The reason given was that what response goes with what stimulus is a relationship that must be remembered in the either facilitating or interfering context of the associations elicited by the stimulus. What is being argued here is that the perceptual response recognition is, so to speak, an $\mathrm{M}$ sensitive switch that permits activation of these associations. It is a switch, moreover, whose off-on status is detectable by the $S$.

\section{REFERENCES}

LAWRENCE, D. H. The nature of the stimulus: Some relationships between learning and perception. In S. Koch (Ed.). Psychology: A study of a science, Vol. 5. New York: McGraw-Hill, 1963. Pp. 179-2:12.

Martin, E. Stimulus pronunciability and aural paired-associate learning. J. verb. Learn. verb. Behav, 1966, 5, 18-22.

Martin, E., and Melton, A. W. Recognition memory for CCC and CVC trigrams of various association values. Human Performance Center, University of Michigan, Tech. Rep. No. 5, 1967.

RroRDAN, J. An introduction to combinatorial analysis. New York: Wiley, 1958.

Shepard, R. N. Comments on Professor Underwood's paper. In C. N. Cofer and B. S. Musgrave (Eds.), Verbal behavior and learning. New York: McGraw-Hill, 1963. Pp. 4870.

Shepard, R. N., and Teghtsoontan, M. Retention of information under conditions approaching a steady state. J. exp. Psychol., 1961, 62, 302 309.

UNDERWOOD, B. J. Stimulus selection in verbal learning. In C. N. Cofer and B. S. Musgrave (Eds.), Verbal behavior and learning. New York: McGraw-Hill, 1963. Pp. 33-48.

WALKER, H. M., AND LEv, I. Statistical inference. New York: Holt, 1953.

WITMER, L. R. The association value of threeplace consonant syllables. J. genet. Psychol. $1935,47,337-360$.

(Received July 12, 1965) 